\title{
Turner syndrome, unicuspid aortopathy, and pregnancy: Difficult decisions for complex scenarios
}

\author{
Síndrome de Turner, aortopatía unicúspide y gestación: decisiones difíciles en escenarios \\ complejos
}

\author{
María Martín ${ }^{1 *}$, Antonio Adeba ${ }^{1 *}$, Jesús M de la Hera ${ }^{1}$, Javier Martínez ${ }^{1}$, Helena Cigarrán², and \\ Rubén Alvarez-Cabo ${ }^{3}$
}

Departments of ${ }^{1}$ Cardiology; ${ }^{2}$ Radiology, and ${ }^{3}$ Cardiac Surgery. Área de Gestión Clínica del Corazón del Hospital Universitario Central de Asturias, Oviedo, Asturias, España

A 31-year-old woman with previous diagnosis of Turner syndrome and desire of pregnancy was submitted for further evaluation for aortic valvulopathy. She was finally diagnosed of unicuspid aortic valve with severe regurgitation and an aortic ascending aneurysm. Given these findings, surgical intervention was decided, with a replacement of the aortic root and ascending aorta by a $34 \mathrm{~mm}$ tube with reimplantation of the aortic valve using the DAVID technique and reconstruction of the unicuspid valve in bicuspid. We review both the unicuspid aortic valve, Turner syndrome, and the recently published European Society of Cardiology guidelines about pregnancy and heart disease. They include a more detailed risk classification that includes different aspects not only maternal and fetal risk but also others such as the periodicity of follow-ups and the right place for delivery, specifically in aortic pathology.

An update of the guidelines of the European Society of Cardiology (ESC) on heart disease and pregnancy was published in $2018^{1,2}$. They include, in relation to the previous ones, several novelties: the World Health Organization (WHO) classification on maternal risk is reinforced; the "heart team" of pregnancy is introduced just like in other pathologies; and greater attention is paid to assisted reproduction therapies that are key in certain pathologies such as Turner syndrome.

\section{Case Report}

With regard to this, we present the case of a 31-yearold patient diagnosed of Turner syndrome and aortic valvulopathy with desire of pregnancy that was referred for assessment and follow-up in our center. The initial transthoracic echocardiogram showed a dilated left ventricle (LV) with borderline systolic function and severe aortic regurgitation. For a more accurate valvular morphology assessment and in the face of surgery, a transesophageal echocardiogram was requested (Figs. $1 \mathrm{~A}$ and $\mathrm{B}$ ) in which a unicuspid aortic valve with severe insufficiency and high gradients in probable relationship with overflow was observed. A dilated and spheroidal LV and aneurysmal dilatation of ascending aorta $\left(46 \mathrm{~mm} \times 45 \mathrm{~mm}\right.$, ASI $\left.>25 \mathrm{~mm} / \mathrm{m}^{2}\right)$ were observed and confirmed in aortic computed tomography scan (Fig. 1C). Given these findings, surgical intervention was decided on, with a replacement of the aortic root and ascending aorta by a $34 \mathrm{~mm}$ tube with reimplantation of the aortic valve using the DAVID technique and reconstruction of the unicuspid valve in

\section{Correspondence:}

*María Martín

E-mail: mmartinf7@ hotmail.com

Date of reception: 22-04-2020

Date of acceptance: 25-05-2020

E-mail: antonio.adeba@gmail.com DOI: 10.24875/ACM.20000176
Available online: 12-04-2021 Arch Cardiol Mex (Eng). 2021;91(2):252-254 www.archivoscardiologia.com 1405-9940 / @ 2020 Instituto Nacional de Cardiología Ignacio Chávez. Published by Permanyer. This is an open access article under the CC BY-NC-ND license (http://creativecommons.org/licenses/by-nc-nd/4.0/). 


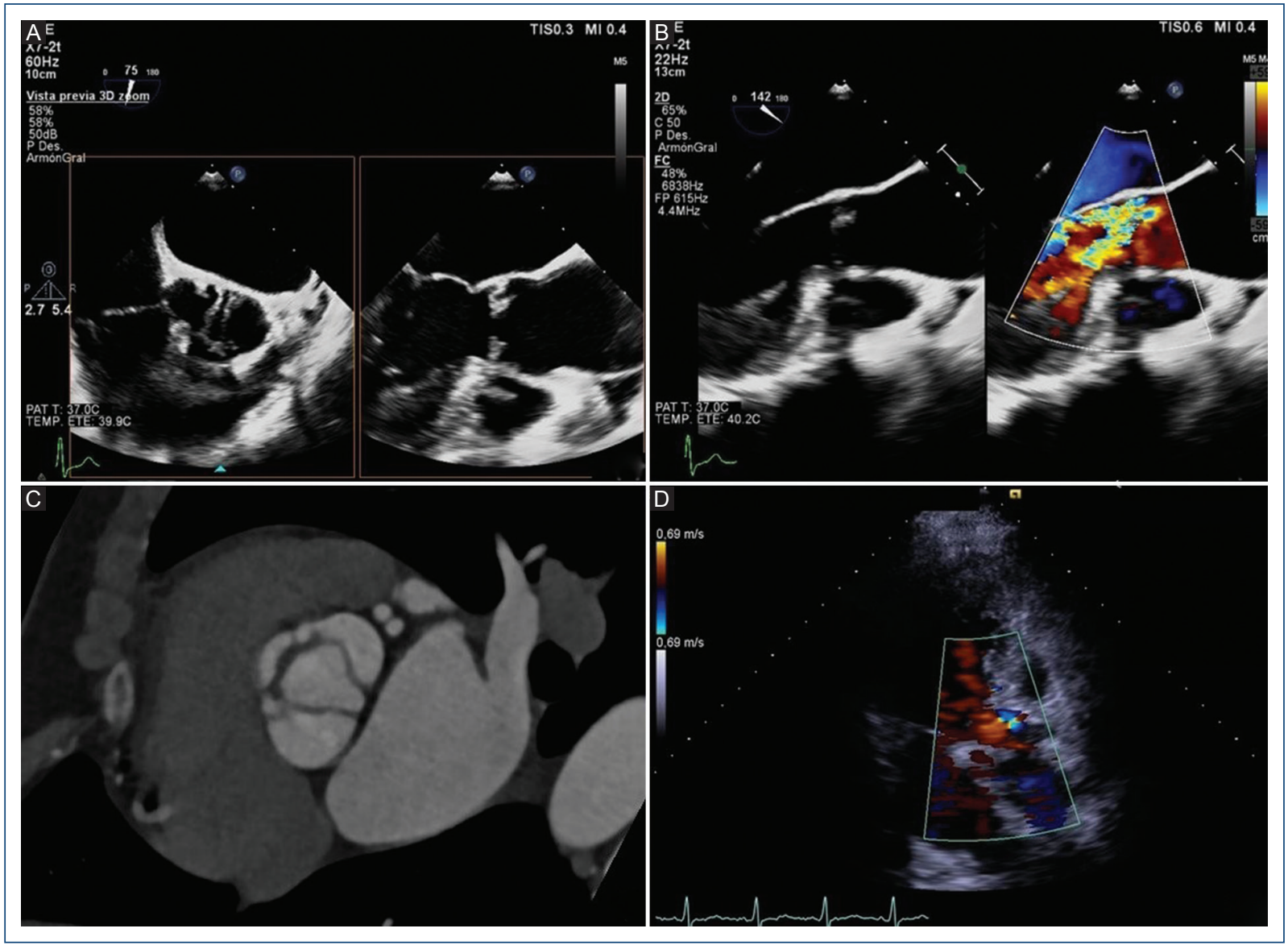

Figure 1. (A) and (B) Transoesophageal echocardiogram showing unicuspid aortic valve with severe regurgitation. (C) Cardiac, computed tomography: unicuspid aortic valve. (D) Transthoracic echocardiogram showing mild aortic regurgitation immediately after surgery.

bicuspid. In the control echo immediately after surgery, minimal aortic insufficiency was observed (Fig. 1D). The post-operative evolution was uneventful. After the surgery, our patient finally dismissed the pregnancy. During the follow-up, she developed progressive degeneration of the pericardial patch used for the valve reconstruction and retraction of valve leaflets requiring at last a new surgery with mechanical prosthesis implantation.

\section{Discussion}

Several aspects are relevant in this case: on the one hand, the unicuspid aortic valve is a rare pathology with an estimated incidence of $0.02 \%$ of the general population and of which isolated cases associated with Turner syndrome have been described. In this, pathology aortic malformations are described in up to $30 \%$ of patients $^{3}$. On the other hand, the possibility of contemplating repairs the valve with replacement of the ascending aorta in the same surgical act avoiding, in this way, the potential complications of a prosthesis, especially in a young woman with desire of pregnancy. Nowadays, thanks to the techniques of assisted reproduction and, especially by ovodonation, gestation is possible in patients with Turner syndrome, taking into account, the high risk of maternal mortality mainly due to cardiovascular diseases and, in particular, due to aortic dissection ${ }^{4}$. In the ESC guidelines that we have previously mentioned, this entity is considered in a precise way, estimating the risk of the patients according to the aortic diameter indexed by the body surface. In our case, given the dilatation of the ascending aorta, pregnancy was initially discouraged due to the high risk of maternal mortality (WHO IV). Even after aortic surgery, there would be a risk of type $B$ dissection due to descending aorta involvement, which, in any case, should be studied and controlled. A periodical and 
multidisciplinary follow-up for these patients is, therefore, essential.

\section{Conclusion}

The new version of the guidelines on pregnancy was necessary to collect new evidence and update recommendations. They include a more detailed risk classification that includes different aspects not only maternal and fetal risk but also others such as the periodicity of follow-ups and the indicated place for delivery, specifically in aortic pathology.

\section{Funding}

There is no financial support on this case, authors do not have any funding about this manuscript.

\section{Conflicts of Interest}

With regard to this manuscript, authors do not have any conflicts of interest.

\section{Ethical Disclosures}

Protection of human and animal subjects. The authors declare that no experiments were performed on humans or animals for this study.

Confidentiality of data. The authors declare that they have followed the protocols of their work center on the publication of patient data.

Right to privacy and informed consent. The authors declare that no patient data appear in this article.

\section{References}

1. Regitz-Zagrosek V, Roos-Hesselink JW, Bauersachs J, Blomström-Lundqvist C, Cífková R, De Bonis M, et al. 2018 ESC Guidelines for the management of cardiovascular diseases during pregnancy Eur Heart $\mathrm{J}$. 2018;39:3165-241.

2. SEC Working Group. ESC guidelines for the management of cardiovascular diseases during pregnancy. Rev Esp Cardiol (Engl Ed) 2019;72: 109-14.

3. Essandoh M, Castellon-Larios K, Zuleta-Alarcon A, Portillo JG, Crestanello JA. Unicuspid aortic valve in a patient with Turner Syndrome. A case report. Front Cardiovasc Med. 2014;1:14.

4. Donadille B, Bernard V, Christin-Maitre S. How can we make pregnancy safe for women with Turner syndrome? Am J Med Genet C Semin Med Genet. 2019;181:100-7. 